Lobo MSC

Silva ACM"

Lins MPE"

Fiszman $\mathbf{R}^{\mathbf{1}}$

Serviço de Epidemiologia e Avaliação. Hospital Universitário Clementino Frag Filho. Universidade Federal do Rio de Janeiro (UFRJ). Rio de Janeiro, RJ, Brasil

Instituto Alberto Luiz Coimbra de PósGraduação e Pesquisa em Engenharia. COPPE. UFRJ. Rio de Janeiro, RJ, Brasil

Correspondência | Correspondence: Maria Stella de Castro Lobo

Serviço de Epidemiologia e Avaliação

Hospital Universitário Clementino Fraga Filho

R. Professor Rodolfo Rocco, $255-5^{\circ}$ andar

Cidade Universitária, Ilha do Fundão

21941-913 Rio de Janeiro, RJ, Brasil

E-mail: clobo@hucff.ufrj.br

\section{Impacto da reforma de} financiamento de hospitais de ensino no Brasil

\section{Impact of the funding reform of teaching hospitals in Brazil}

\section{RESUMO}

OBJETIVO: Avaliar o impacto da reforma de financiamento na produtividade de hospitais de ensino.

MÉTODOS: A partir do Sistema de Informações dos Hospitais Universitários Federais, foram construídas fronteiras de eficiência e produtividade em 2003 e 2006 com técnicas de programação linear, por meio de análise envoltória de dados, considerando retornos variáveis de escala e orientação a input. Calculouse o Índice de Malmquist para identificar mudanças de desempenho ao longo dos anos quanto à eficiência técnica (razão entre os escores de eficiência em tempos distintos) e eficiência tecnológica (deslocamento da fronteira no período considerado).

RESULTADOS: Houve aumento do aporte financeiro em $51 \%$ e da eficiência técnica dos hospitais de ensino (de 11, passaram a ser 17 na fronteira empírica de eficiência), o mesmo não ocorrendo com a fronteira tecnológica. O uso de análise envoltória de dados estabeleceu os benchmarks para as unidades ineficientes (antes e depois da reforma) e os escores de eficiência mostraram uma possível correlação entre a eficiência técnica encontrada e a intensidade e dedicação de ensino.

CONCLUSÕES: A reforma permitiu o desenvolvimento de melhorias gerenciais, mas é necessário maior tempo de acompanhamento para observar mudanças mais efetivas do modelo de financiamento.

DESCRITORES: Hospitais de Ensino, organização \& administração. Custos e Análise de Custo. Taxas, Razões e Proporções, métodos. Administração Financeira de Hospitais. Economia Hospitalar. Eficiência Organizacional. 


\begin{abstract}
OBJECTIVE: To assess the impact of funding reform on the productivity of teaching hospitals.

METHODS: Based on the Information System of Federal University Hospitals of Brazil, 2003 and 2006 efficiency and productivity were measured using frontier methods with a linear programming technique, data envelopment analysis, and input-oriented variable returns to scale model. The Malmquist index was calculated to detect changes during the study period: "technical efficiency change," or the relative variation of the efficiency of each unit; and "technological change" after frontier shift.

RESULTS: There was $51 \%$ mean budget increase and improvement of technical efficiency of teaching hospitals (previously 11, 17 hospitals reached the empirical efficiency frontier) but the same was not seen for the technology frontier. Data envelopment analysis set benchmark scores for each inefficient unit (before and after reform) and there was a positive correlation between technical efficiency and teaching intensity and dedication.
\end{abstract}

CONCLUSIONS: The reform promoted management improvements but there is a need of further follow-up to assess the effectiveness of funding changes.

DESCRIPTORS: Hospitals, Teaching, organization \& administration. Costs and Cost Analysis. Rates, Ratios and Proportions, methods. Financial Management, Hospital. Economics, Hospital. Efficiency, Organizational.

\title{
INTRODUÇÃO
}

Os hospitais universitários e de ensino (HUE) têm papel de relevância na atenção de saúde no Brasil, respondendo por $10 \%$ dos leitos, $12 \%$ das internações e da produção ambulatorial, $26 \%$ dos leitos de unidade de tratamento intensivo (UTI), $38 \%$ dos procedimentos de alta complexidade ofertados pelo Sistema Único de Saúde (SUS). ${ }^{11}$

Ainda assim, nos últimos anos, tem sido colocada em pauta a crise dos hospitais no Brasil e, em particular, a dos hospitais de ensino, que perpassa elementos de financiamento e de gestão, implicando queda de desempenho dos seus respectivos modelos assistenciais, de ensino e de pesquisa.

Como primeira estratégia para melhoria da situação financeira, foi instituída Comissão Interinstitucional, em 2003, com a participação dos Ministérios da Saúde, Educação, Ciência e Tecnologia e do Planejamento, presidida pelo primeiro, com o objetivo de avaliar a situação dos HUE, visando a reorientar a política nacional para o setor. ${ }^{a}$ Esta avaliação mostrou a necessidade de maior integração dos hospitais de ensino aos sistemas locais de saúde, além de indicar que os hospitais sofriam de déficit de financiamento e careciam de melhoria nas capacidades de gestão de recursos.

A partir do diagnóstico inicial, foram definidos os instrumentos e estabelecidos os pré-requisitos para a certificação dos hospitais de ensino do País, baseados nos eixos de: integração docente-assistencial, participação na rede de serviços do SUS e manutenção de mecanismos gerenciais e organizativos. ${ }^{b}$ Uma vez certificados, os hospitais passaram a ser incluídos no Programa de Reestruturação de Hospitais de Ensino no âmbito do SUS e iniciaram processo de pactuação, com os respectivos gestores de saúde, de metas relacionadas à produção de serviços $\mathrm{e}$ às atividades de ensino, de pesquisa e de avaliação tecnológica voltadas para as necessidades do sistema de saúde. Por meio da contratualização, o mecanismo de financiamento dos HUE foi definitivamente alterado,

a Ministério da Educação, Ministério da Saúde, Ministério da Ciência e Tecnologia e Ministério de Planejamento, Orçamento e Gestão Portaria Interministerial 562 de 12 de maio 2003. Institui Comissão Interinstitucional com o objetivo de avaliar e diagnosticar a situação dos HUEs no Brasil, visando reformular e/ou reorientar a política nacional para o setor, considerando a necessidade de: melhoria da situação financeira, estrutural, organizativa e de gestão e definição do papel / inserção no SUS. Diário Oficial da União. 28 maio 2003 ; Seção II: p.23. ${ }^{b}$ Ministério da Educação, Ministério da Saúde. Portaria Interministerial 1.000, de 15 de abril de 2004. Dispõe sobre os requisitos necessários para a certificação dos hospitais de ensino. Diário Oficial da União. 15 abril 2004; Seção I: p.13-4. 
baseado em orçamento global para os procedimentos de média complexidade, de acordo com o cumprimento das metas pactuadas. A alta complexidade continua a ser paga de acordo com a produção. ${ }^{9}$

O novo financiamento baseado no orçamento fixo e cumprimento de metas acordadas teve tripla orientação: a) adequação entre a demanda do sistema de saúde e a oferta de serviços assistenciais, de formação de recursos humanos e de desenvolvimento de pesquisa; $b$ ) aumento do aporte financeiro, principal reivindicação dos diretores das unidades; c) garantia de esforços para o desenvolvimento da capacidade de administração e gestão local, principal ótica dos órgãos federais responsáveis pelo repasse financeiro.

Considerando que uma das principais razões para a reforma consistiu na necessidade de redução das ineficiências da atenção de saúde prestada pelos hospitais de ensino, o objetivo do presente estudo foi avaliar o impacto de uma reforma financeira na produtividade de hospitais de ensino. Buscou-se também diferenciar o componente tecnológico da mudança de mecanismo de transferência de recursos, enquanto instrumento de política pública nacional, daquele inerente à própria capacidade de cada unidade para gerenciar a nova forma de alocação financeira.

\section{MÉTODOS}

A medida de eficiência em análise envoltória de dados (DEA, do inglês data envelopment analysis), baseada em programação linear, é realizada por meio da comparação de um conjunto de unidades similares, denominadas em inglês decision making units (DMU), as quais consomem os mesmos inputs (recursos) para produzir os mesmos outputs (produtos), diferenciando-se unicamente nas quantidades consumidas e produzidas. Assim, uma DMU será eficiente se, comparativamente às demais, tiver maior produção para quantidades fixas de recursos e/ou utilizar menos recursos para gerar uma quantidade fixa de produtos.

Ao definir as DMU com as melhores práticas, a DEA constrói uma fronteira de produção empírica eficiente. As DMU que se encontram sobre a fronteira, portanto, eficientes, têm medida de eficiência de $100 \%$, enquanto as DMU localizadas abaixo da fronteira são ineficientes. A medida de eficiência das DMU ineficientes pode ser calculada pela distância "radial" da fronteira, conhecida como eficiência técnica, que indica a proporção em que devem ser reduzidos os inputs, ou aumentados os outputs, para que a fronteira eficiente seja alcançada. ${ }^{5}$

A DEA também tem por objetivo indicar onde surgem as ineficiências das DMU e apresentar uma referência ou benchmark para que as unidades ineficientes possam atingir a fronteira de eficiência técnica.
O modelo de produção usado foi o DEA BCC (nomeado pelas iniciais dos autores Banker, Charnes e Cooper $^{1}$ ) orientado à redução de input para projeção na fronteira. O modelo de programação linear DEA $\mathrm{BCC}$ (equação 1) considera retornos variáveis de escala visto que alguns estudos já indicaram a existência de economias de escala na eficiência hospitalar. ${ }^{13,16} \mathrm{O}$ uso de retornos variáveis de escala permite que uma unidade eficiente somente seja comparada com unidades produtivas de tamanho similar em escala semelhante. ${ }^{3}$ Este aspecto é relevante quando se pretende utilizar o conhecimento dos benchmarks na pactuação entre gestor e unidades de saúde.

Da mesma forma, a escolha de orientação a input se deve ao fato de que os gestores hospitalares têm maior controle sobre os inputs do que sobre os outputs e, na maior parte dos países, a ênfase da administração em saúde está no maior controle de custos, sem prejuízo da qualidade, em vez de se priorizar o aumento da demanda. Isso explica o porquê de a maioria dos estudos que utilizam DEA para aferição da eficiência em hospitais ser orientada a input, conforme observado em revisão da literatura. ${ }^{14}$

$$
\min h
$$

$$
\begin{aligned}
\text { tal que } h \mathrm{x} & \geq \sum \lambda_{\mathrm{j}} \mathrm{x}_{\mathrm{j}} \\
\mathrm{y} & \leq \sum \lambda_{\mathrm{j}} \mathrm{y}_{\mathrm{j}} \\
\lambda_{\mathrm{j}} & \geq 0 \quad \forall_{\mathrm{j}} \quad \text { equação 1 } \\
\mathrm{h} & \geq 0 \\
\sum_{\mathrm{j}=1}^{\mathrm{n}} \lambda_{\mathrm{j}} & =1 \\
\lambda_{\mathrm{j}} & \geq 0 \quad \forall \mathrm{j}
\end{aligned}
$$

O índice de Malmquist mede a variação da produtividade em períodos distintos de tempo. Este índice foi desenvolvido por Caves et $a 1,{ }^{2}$ inspirados no trabalho de Malmquist. ${ }^{12}$ Quando se comparam dois períodos de tempo, observam-se duas fronteiras distintas: a do período inicial $(\mathrm{t})$ e a do período final $(\mathrm{t}+1)$. Portanto, é possível obter índices de desempenho de acordo com a tecnologia de referência (fronteira) assumida (antes e depois).

O método de Malmquist-DEA, proposto por Färe et al, ${ }^{4}$ tem sido o mais amplamente aplicado para o cálculo da medida. O método aplica o algoritmo de programação linear de DEA para construção da fronteira de produção em ambos os períodos analisados e considera a distância de cada hospital ou DMU considerada $\left(\mathrm{DMU}_{0}\right)$, antes e depois, às duas fronteiras distintas. $\mathrm{O}$ índice de Malmquist-DEA $\left(\mathrm{M}_{\mathrm{o}}\right)$ é calculado pela média geométrica de dois índices, no qual o primeiro utiliza como referência a fronteira do período t e o segundo a 
fronteira do período $t+1$. Um valor de $\mathrm{M}_{0}$ maior que 1 indica um crescimento ou evolução da produtividade total dos fatores (PTF) entre os períodos t e $t+1$, enquanto que um valor menor que 1 indica um declínio.

O índice de Malmquist orientado a input é dado por:

$M_{0}=\left[\frac{D_{0}^{t}\left(x_{0}^{t+1}, y_{0}^{t+1}\right)}{D_{0}^{t}\left(x_{0}^{t}, y_{0}^{t}\right)} \frac{D_{0}^{t+1}\left(x_{0}^{t+1}, y_{0}^{t+1}\right)}{D_{0}^{t+1}\left(x_{0}^{t}, y_{0}^{t}\right)}\right]^{1 / 2}$

Onde:

$D_{0}^{t}\left(x_{0}^{t}, y_{0}^{t}\right)$ corresponde à medida de eficiência técnica da $\mathrm{DMU}_{0}$ no período t, obtida com as observações de todas as DMU no período t, ou seja, $D_{0}^{t}\left(x_{0}^{t}, y_{0}^{t}\right)=\theta_{0}^{t}$,

$D_{0}^{t+1}\left(x_{0}^{t+1}, y_{0}^{t+1}\right)$ corresponde à medida de eficiência técnica da $\mathrm{DMU}_{0}$ no período $\mathrm{t}+1$, obtida com as observações de todas as DMU no período $\mathrm{t}+1$, ou seja, $D_{0}^{t+1}\left(x_{0}^{t+1}, y_{0}^{t+1}\right)=\theta_{0}^{t+1}$,

$D_{0}^{t}\left(x_{0}^{t+1}, y_{0}^{t+1}\right)$ corresponde à medida de eficiência técnica da $\mathrm{DMU}_{0}$ obtida ao substituir os dados da $\mathrm{DMU}_{0}$ no período $t$ por aqueles do período $t+1$, desde que as observações das demais DMU tenham sido realizadas no período $t$,

$D_{0}^{t+1}\left(x_{0}^{t}, y_{0}^{t}\right)$ corresponde à medida de eficiência técnica da $\mathrm{DMU}_{0}$ obtida ao substituir os dados da $\mathrm{DMU}_{0}$ no período $t+1$ por aqueles do período $t$, desde que as observações das demais DMU tenham sido realizadas no período $\mathrm{t}+1$.

Em sua forma decomposta, o índice de Malmquist permite separar as fontes de variação de produtividade em dois termos:

$M_{o}\left(x_{t+1}, y_{t+1}, x_{t}, y_{t}\right)=\frac{d_{o}^{t+1}\left(x_{t+1}, y_{t+1}\right)}{d_{o}^{t}\left(x_{t}, y_{t}\right)} *\left[\left(\frac{d_{o}^{t}\left(x_{t+1}, y_{t+1}\right)}{d_{o}^{t+1}\left(x_{t+1}, y_{t+1}\right)}\right) *\left(\frac{d_{o}^{t}\left(x_{t}, y_{t}\right)}{d_{o}^{t+1}\left(x_{t}, y_{t}\right)}\right]^{1 / 2}\right.$

Nesta decomposição, a razão fora dos parênteses mede a mudança na eficiência relativa da unidade observadae é chamada de "mudança técnica" ( catch-up). Esta razão indica se a produção da unidade está se aproximando ou se afastando da fronteira. Enquanto isso, a média geométrica das duas razões dentro dos parênteses mede a mudança da fronteira tecnológica entre os dois perí-

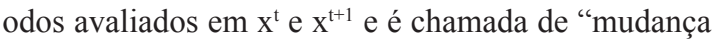
na eficiência tecnológica" (frontier shift). Estes índices parciais são importantes porque permitem verificar se um eventual aumento da eficiência relativa de um período para o outro deve ser atribuído ao aumento da produtividade da unidade avaliada ou à contração da fronteira de produção.

A mudança técnica (catch-up) representa o aumento da eficiência decorrente da maior injeção de recursos financeiros (razão entre o escore de eficiência em 2006 e em 2003, independentemente do comportamento da fronteira). A alteração tecnológica é diretamente decorrente do deslocamento da fronteira (frontier shift), no caso, a partir da introdução de um novo modelo de gestão. Os termos do índice, portanto, guardam uma equivalência de discurso entre a formulação matemática e a visão dos atores da reforma, sejam diretores de unidade (que enfatizam a crise de financiamento) ou gestores responsáveis pelo financiamento (que buscam o enfrentamento da crise de gestão).

Os dados analisados são provenientes do Sistema de Informação dos Hospitais Universitários Federais do Ministério da Educação (SIHUF/MEC), referentes ao segundo semestre de 2003 e segundo semestre de 2006, portanto, antes e depois da reforma por contratualização. De modo a garantir um grupo homogêneo para comparação, foram excluídos os hospitais de especialidade e as maternidades. Os 30 hospitais gerais do MEC correspondem à cerca de $20 \%$ dos hospitais de ensino do País e estão representados pelas siglas das respectivas universidades.

Seguindo modelo proposto por Ozcan, ${ }^{15}$ foram usadas como variáveis de inputs: despesas operacionais (valor baseado no total de entrada mensal de recursos SUS, antes e depois do contrato de metas, visto que essa é a principal fonte de custeio desses hospitais); força de trabalho (número total de médicos e de não médicos), número de leitos e mix de serviços. Segundo o autor, as duas últimas são variáveis proxies satisfatórias para o volume de capital do hospital (correlação de Pearson= 0,926 ). O mix de serviços denota o número total de serviços diagnósticos e terapêuticos ofertados pela unidade e, portanto, a complexidade dos serviços.

Como outputs, foram consideradas: admissões, cirurgias e consultas ambulatoriais, todas ajustadas de acordo com o índice de complexidade do hospital. Também como medida proxy de case mix, o SIHUF inclui uma variável contínua denominada Sistema de Informações de Procedimentos de Alta Complexidade (SIPAC), baseada no volume de recursos necessários para a realização dos procedimentos de alta complexidade credenciados pelo Ministério da Saúde, tais como neurocirurgias, cirurgias cardíacas, ortopédicas, transplantes, entre outros. Para o ajuste, dividiu-se cada valor individual de SIPAC pela média de valores do conjunto de hospitais, criando-se o índice SIPAC, então multiplicado por cada variável de output. ${ }^{10}$

Finalmente, para avaliação da dimensão de ensino dos hospitais, foram usados os seguintes indicadores, conforme Grosskopf et al: ${ }^{7}$ intensidade de ensino (relação residentes/leitos) e dedicação de ensino (relação residentes/médicos). 


\section{RESULTADOS}

Com a reforma do financiamento, a receita dos hospitais de ensino proveniente do SUS passou a incorporar: os valores contratualizados de média complexidade, os produzidos de alta complexidade, além de valores de incentivo (como os de contratualização, de inserção no sistema de emergência, oferta de UTI, entre outros). Isso implicou incremento médio de receita em 51\% (Tabela 1), sem que ocorresse para esses hospitais aumento

Tabela 1. Variação da receita Sistema Único de Saúde e da produção assistencial dos hospitais estudados antes e depois da reforma financeira. Brasil, 2003-2006.

\begin{tabular}{|c|c|c|c|c|c|c|}
\hline Hospital & $\begin{array}{l}\text { Receita SUS } 2003 \\
2003\left(\times 10^{6}\right)\end{array}$ & $\begin{array}{l}\text { Receita SUS } 2006 \\
2006\left(\times 10^{6}\right)\end{array}$ & $\begin{array}{c}\text { Diferença (\%) } \\
\text { Receita }\end{array}$ & $\begin{array}{c}\text { Diferença (\%) } \\
\text { Cirurgias }\end{array}$ & $\begin{array}{c}\text { Diferença (\%) } \\
\text { Admissões }\end{array}$ & $\begin{array}{c}\text { Diferença (\%) } \\
\text { Consultas }\end{array}$ \\
\hline FMTM & 1,54 & 2,31 & 49,0 & $-7,0$ & 51,0 & 36,0 \\
\hline FUAM & 0,71 & 0,53 & $-26,0$ & 0,0 & $-14,0$ & 4,0 \\
\hline FUFMS & 1,03 & 1,37 & 33,0 & 33,0 & 1,0 & 15,0 \\
\hline FUFS & 0,19 & 0,35 & 87,0 & 133,0 & 143,0 & 162,0 \\
\hline FURG & 0,54 & 0,76 & 40,0 & $-28,0$ & $-2,0$ & 211,0 \\
\hline HCPA & 5,35 & 6,65 & 24,0 & 10,0 & 2,0 & 40,0 \\
\hline UFAL & 1,01 & 0,96 & $-6,0$ & 4,0 & $-15,0$ & 9,0 \\
\hline UFBA & 0,95 & 1,89 & 100,0 & 24,0 & 24,0 & 5,0 \\
\hline UFCE & 1,43 & 3,36 & 136,0 & $-21,0$ & 0,0 & 10,0 \\
\hline UFCG & 0,33 & 0,33 & $-2,0$ & 0,0 & $-41,0$ & 7,0 \\
\hline UFES & 1,31 & 1,52 & 16,0 & $-11,0$ & $-3,0$ & $-15,0$ \\
\hline UFF & 1,47 & 1,92 & 30,0 & $-5,0$ & 6,0 & 75,0 \\
\hline UFGO & 2,02 & 2,60 & 29,0 & 23,0 & 10,0 & 89,0 \\
\hline UFJF & 0,45 & 0,65 & 45,0 & 120,0 & 84,0 & 134,0 \\
\hline UFMA & 3,03 & 4,42 & 46,0 & 15,0 & 11,0 & 72,0 \\
\hline UFMG & 3,74 & 4,81 & 29,0 & 8,0 & $-2,0$ & 44,0 \\
\hline UFMT & 0,51 & 0,92 & 80,0 & 76,0 & 52,0 & 167,0 \\
\hline UFPA & 0,52 & 1,72 & 229,0 & 110,0 & 71,0 & 192,0 \\
\hline UFPB & 0,43 & 0,78 & 80,0 & 18,0 & 39,0 & 70,0 \\
\hline UFPE & 1,48 & 2,19 & 48,0 & $-2,0$ & 9,0 & 81,0 \\
\hline UFPEL & 0,81 & 1,24 & 54,0 & $-11,0$ & $-23,0$ & 17,0 \\
\hline UFPR & 3,62 & 5,65 & 56,0 & 10,0 & 14,0 & 69,0 \\
\hline UFRJ & 2,42 & 3,98 & 65,0 & $-28,0$ & $-10,0$ & 4,0 \\
\hline UFRN & 0,53 & 0,98 & 86,0 & $-20,0$ & $-15,0$ & $-12,0$ \\
\hline UFSC & 1,09 & 1,98 & 81,0 & 0,0 & 17,0 & 121,0 \\
\hline UFSM & 1,90 & 2,62 & 38,0 & 6,0 & 19,0 & 104,0 \\
\hline UFU & 3,95 & 4,85 & 23,0 & $-20,0$ & $-11,0$ & 72,0 \\
\hline UNB & 1,25 & 1,92 & 54,0 & $-27,0$ & $-61,0$ & $-1,0$ \\
\hline UNI-RIO & 0,67 & 0,74 & 10,0 & 20,0 & $-11,0$ & 3,0 \\
\hline UNIFESP & 10,24 & 9,80 & $-4,0$ & $-33,0$ & $-10,0$ & 39,0 \\
\hline Média & 1,82 & 2,46 & 51,0 & $-6,0$ & $-4,0$ & 44,0 \\
\hline
\end{tabular}

Fonte: Sistema de Informações dos Hospitais Universitários Federais (SIHUF).

FMTM: Faculdade de Medicina do Triângulo Mineiro; FUAM: Fundação Universitária do Amazonas; FUFMS: Fundação Universitária de Mato Grosso do Sul; FUFS: Fundação Universitária de Sergipe; FURG: Fundação Universitária de Rio Grande; HCPA: Hospital das Clínicas de Porto Alegre; UFAL: Universidade Federal de Alagoas; UFBA: Universidade Federal da Bahia; UFCE: Universidade Federal do Ceará; UFCG: Universidade Federal de Campina Grande; UFES: Universidade Federal do Espírito Santo; UFF: Universidade Federal Fluminense; UFGO: Universidade Federal de Goiás; UFJF: Universidade Federal de Juiz de Fora; UFMA: Universidade Federal do Maranhão; UFMG: Universidade Federal de Minas Gerais; UFMT: Universidade Federal de Mato Grosso; UFPA: Universidade Federal do Pará; UFPB: Universidade Federal da Paraíba; UFPE: Universidade Federal de Pernambuco; UFPEL: Universidade Federal de Pelotas; UFPR: Universidade Federal do Paraná; UFRJ: Universidade Federal do Rio de Janeiro; UFRN: Universidade Federal do Rio Grande do Norte; UFSC: Universidade Federal de Santa Catarina; UFSM: Universidade Federal de Santa Maria; UFU: Universidade Federal de Uberlândia; UNB: Universidade de Brasília; UNI-RIO: Universidade do Rio de Janeiro; UNIFESP: Universidade Federal de São Paulo. 
Tabela 2. Eficiência dos hospitais e Índice de Malmquist/DEA decomposto. Brasil, 2003-2006.

\begin{tabular}{|c|c|c|c|c|c|}
\hline Hospital & Eficiência 2003 & Eficiência 2006 & Malmquist & Catch-up & Frontier shift \\
\hline FMTM & 0,87 & 1,00 & 0,96 & 1,15 & 0,84 \\
\hline FUAM & 0,88 & 1,00 & 0,98 & 1,13 & 0,87 \\
\hline FUFMS & 0,68 & 0,76 & 0,92 & 1,12 & 0,82 \\
\hline FUFS & 1,00 & 1,00 & 0,74 & 1,00 & 0,74 \\
\hline$F_{U R G}{ }^{a}$ & 0,96 & 0,95 & 0,97 & 0,99 & 0,98 \\
\hline HCPA & 1,00 & 1,00 & 0,96 & 1,00 & 0,96 \\
\hline UFAL & 0,83 & 0,92 & 0,97 & 1,14 & 0,85 \\
\hline$U_{F B A}{ }^{a}$ & 0,83 & 0,63 & 0,74 & 0,76 & 0,97 \\
\hline UFCE ${ }^{\mathbf{a}}$ & 0,80 & 0,67 & 0,81 & 0,84 & 0,97 \\
\hline UFCG & 0,95 & 1,00 & 0,81 & 0,98 & 0,83 \\
\hline UFES & 0,93 & 0,90 & 0,82 & 1,03 & 0,80 \\
\hline UFF & 0,75 & 0,84 & 0,99 & 1,12 & 0,88 \\
\hline UFGO & 0,80 & 1,00 & 1,02 & 1,25 & 0,82 \\
\hline UFJF & 1,00 & 1,00 & 0,99 & 1,00 & 0,99 \\
\hline UFMA & 0,72 & 0,83 & 0,96 & 1,15 & 0,84 \\
\hline UFMG & 1,00 & 1,00 & 0,93 & 1,00 & 0,93 \\
\hline UFMT & 1,00 & 1,00 & 0,93 & 1,00 & 0,93 \\
\hline UFPA & 1,00 & 1,00 & 0,96 & 1,00 & 0,96 \\
\hline UFPB & 0,99 & 1,00 & 0,96 & 1,01 & 0,95 \\
\hline UFPE & 0,81 & 1,00 & 0,85 & 1,24 & 0,69 \\
\hline UFPEL & 1,00 & 1,00 & 0,93 & 1,00 & 0,93 \\
\hline UFPR & 0,90 & 1,00 & 0,93 & 1,11 & 0,84 \\
\hline UFRJ & 1,00 & 1,00 & 0,65 & 1,00 & 0,65 \\
\hline UFRN ${ }^{a}$ & 0,84 & 0,56 & 0,58 & 0,67 & 0,87 \\
\hline UFSC & 0,63 & 0,68 & 0,84 & 1,08 & 0,78 \\
\hline UFSM & 0,81 & 0,90 & 0,92 & 1,11 & 0,83 \\
\hline UFU ${ }^{\mathbf{b}}$ & 1,00 & 1,00 & - & 1,00 & - \\
\hline$U_{N B^{a}}$ & 1,00 & 0,98 & 0,47 & 0,98 & 0,47 \\
\hline UNI-RIO ${ }^{\mathbf{a}}$ & 0,91 & 0,88 & 0,96 & 0,99 & 0,97 \\
\hline UNIFESPb & 1,00 & 1,00 & - & 1,00 & - \\
\hline Média & 0,90 & 0,92 & 0,88 & 1,03 & 0,86 \\
\hline
\end{tabular}

${ }^{\text {a }}$ Hospitais que tiveram queda de eficiência técnica no período.

bInviabilidade do modelo decorrente da projeção dos dados de 2006 na fronteira de 2003.

concomitante do número de internações e cirurgias ajustadas (queda de $6 \%$ e $4 \%$, respectivamente), mas havendo acréscimo das consultas de pacientes externos (44\%). As demais variáveis de input não sofreram mudanças significativas no período, como o número de leitos e de funcionários, e mix de serviços.

Na Tabela 2, são apresentadas as eficiências relativas dos hospitais de acordo com as fronteiras BCC-Input nos anos de 2003 e de 2006. Em 2003, havia 11 hospitais eficientes (cinco deles na região mais produtiva de escala - RMPE) e esse número alcançou 17 unidades em 2006 (11 na RMPE). No mesmo período, a média de eficiência aumentou de $89,6 \%$ para $91,7 \%$. Conside- radas as fronteiras separadamente, apenas seis unidades tiveram redução das suas eficiências relativas - FURG, UFBA, UFCE, UFRN, UnB e UNI-RIO - apesar do aumento de suas receitas no período em, respectivamente, $40 \%, 100 \%, 136 \%, 86 \%, 54 \%, 10 \%$. Entre essas unidades, somente a UFBA não teve queda em nenhuma categoria de produção/output (Tabela 1).

Apenas a UFGO teve valor da medida Índice de Malmquist superior a 1,0 (sua produção de cirurgias aumentou em $23 \%$; de internações, em $10 \%$; e de consultas, em 89\%). Na análise do Índice de Malmquist decomposto, observou-se, por outro lado, aumento ou igualdade da eficiência técnica (catch-up) em 23 dos 
Tabela 3. Benchmarks para os hospitais estudados. Brasil, 2003-2006.

\begin{tabular}{|c|c|c|}
\hline Hospital & $\begin{array}{c}2003 \\
\text { Benchmarks }\end{array}$ & $\begin{array}{c}2006 \\
\text { Benchmarks }\end{array}$ \\
\hline FMTM & $\begin{array}{l}\text { FUFS, UFRJ, UFU, } \\
\text { UNB, UNIFESP }\end{array}$ & Eficiente \\
\hline FUAM & $\begin{array}{c}\text { FUFS, UFJF, UFRJ, } \\
\text { UFU, }\end{array}$ & Eficiente \\
\hline FUFMS & $\begin{array}{l}\text { UFJF, UFRJ, UFU, } \\
\text { UB, UNIFESP }\end{array}$ & $\begin{array}{l}\text { UFCG, UFJF, UFPE, } \\
\text { UFRJ }\end{array}$ \\
\hline FUFS & Eficiente & Eficiente \\
\hline FURG & $\begin{array}{c}\text { FUFS, UFJF, } \\
\text { UFPEL, UFU, UNB }\end{array}$ & $\begin{array}{c}\text { FUFS, UFJF, UFPEL, } \\
\text { UNIFESP }\end{array}$ \\
\hline HCPA & Eficiente & Eficiente \\
\hline UFAL & $\begin{array}{c}\text { FUFS, UFJF, } \\
\text { UFPEL, UFU, UNB }\end{array}$ & $\begin{array}{c}\text { FMTM, FUAM, FUFS, } \\
\text { UFGO, UFJF }\end{array}$ \\
\hline UFBA & $\begin{array}{c}\text { FUFS, UFJF, UFRJ, } \\
\text { UNIFESP }\end{array}$ & $\begin{array}{c}\text { FUFS, UFJF, UFMG, } \\
\text { UNIFESP }\end{array}$ \\
\hline UFCE & $\begin{array}{c}\text { FUFS, UFRJ, UFU, } \\
\text { UNIFESP }\end{array}$ & $\begin{array}{l}\text { HCPA, UFJF, UFMG, } \\
\text { UFPEL, UNIFESP }\end{array}$ \\
\hline UFCG & $\begin{array}{l}\text { FUFS, UFPA, } \\
\text { UNB, UNIFESP }\end{array}$ & Eficiente \\
\hline UFES & $\begin{array}{c}\text { FUFS, UFRJ, UFU, } \\
\text { UNIFESP }\end{array}$ & $\begin{array}{c}\text { FUAM, HCPA, UFGO, } \\
\text { UFJF }\end{array}$ \\
\hline UFF & $\begin{array}{l}\text { FUFS, UFRJ, } \\
\text { UNIFESP }\end{array}$ & $\begin{array}{c}\text { FUAM, UFGO, UFJF, } \\
\text { UNIFESP }\end{array}$ \\
\hline UFGO & $\begin{array}{c}\text { FUFS, UFRJ, UFU, } \\
\text { UNIFESP }\end{array}$ & Eficiente \\
\hline UFJF & Eficiente & Eficiente \\
\hline UFMA & $\begin{array}{l}\text { FUFS, UFRJ, UFU, } \\
\text { UNB, UNIFESP }\end{array}$ & HCPA, UFJF, UFU \\
\hline UFMG & Eficiente & Eficiente \\
\hline UFMT & Eficiente & Eficiente \\
\hline UFPA & Eficiente & Eficiente \\
\hline UFPB & $\begin{array}{c}\text { FUFS, UFPA, } \\
\text { UFRJ, UNB, } \\
\text { UNIFESP }\end{array}$ & Eficiente \\
\hline UFPE & $\begin{array}{l}\text { FUFS, UFRJ, UFU, } \\
\text { UNB, UNIFESP }\end{array}$ & Eficiente \\
\hline UFPEL & Eficiente & Eficiente \\
\hline UFPR & $\begin{array}{l}\text { UFJF, UFRJ, UFU, } \\
\text { UNB, UNIFESP }\end{array}$ & Eficiente \\
\hline UFRJ & Eficiente & Eficiente \\
\hline UFRN & $\begin{array}{l}\text { FUFS, UFRJ, } \\
\text { UNIFESP }\end{array}$ & $\begin{array}{c}\text { FMTM, FUAM, FUFS, } \\
\text { UFJF, UNIFESP }\end{array}$ \\
\hline UFSC & $\begin{array}{l}\text { FUFS, UFJF, UFRJ, } \\
\text { UFU, UNB }\end{array}$ & $\begin{array}{l}\text { FMTM, FUFS, UFJF, } \\
\text { UFPEL, UNIFESP }\end{array}$ \\
\hline UFSM & $\begin{array}{l}\text { FUFS, UFJF, } \\
\text { UFPEL, UFU }\end{array}$ & $\begin{array}{l}\text { HCPA, UFJF, UFMG, } \\
\text { UFPEL, UFU }\end{array}$ \\
\hline UFU & Eficiente & Eficiente \\
\hline UNB & Eficiente & $\begin{array}{l}\text { UFCG, UFJF, UFPE, } \\
\text { UFRJ }\end{array}$ \\
\hline UNI-RIO & $\begin{array}{l}\text { FUFS, UFJF, UFRJ, } \\
\text { UNIFESP }\end{array}$ & $\begin{array}{c}\text { FUAM, UFCG, UFJF, } \\
\text { UNIFESP }\end{array}$ \\
\hline UNIFESP & Eficiente & Eficiente \\
\hline
\end{tabular}

hospitais. Entre aqueles eficientes em 2003, somente a UnB se afastou da fronteira eficiente. Com relação à eficiência tecnológica, todos os valores aferidos tiveram valor inferior a 1,0, o que significa contração da fronteira. As inviabilidades do modelo encontradas para UFU e UNIFESP foram decorrentes de a localização geométrica de 2006 estar acima da fronteira de 2003, portanto, fora do conjunto de possibilidades de produção para aquele período.

A Tabela 3 mostra, em ambos os períodos estudados, a relação dos hospitais de referência para cada unidade ineficiente. Mesmo aferido por modelagem matemática, isto é, pelo cálculo da menor distância à fronteira em que os hospitais de referência serão vértices de um poliedro da fronteira empírica, o modelo garantiu similaridades entre os pares de comparação: porte, geográfica, características e vocações dos serviços prestados. Para atingir a fronteira, os hospitais ineficientes podem estudar os valores absolutos das variáveis de seus benchmarks, para que possíveis mudanças sejam sinalizadas.

Considerando a redução de inputs necessária para que todos os hospitais atingissem a fronteira, em 2003, a mesma seria de $13 \%$ (7.299) dos funcionários, 19\% (1.448) dos médicos, $19 \%$ (1.651) dos leitos, $25 \%$ do mix de serviços, além de $8,3 \%$ ou $\mathrm{R} \$ 4.600 .000$ nas despesas operacionais. Em 2006, o decréscimo necessário seria de: $9 \%$ dos funcionários (5.060), 16\% dos médicos (1.251), 9\% dos leitos (740), 17\% do mix de serviços ofertados. Quanto às despesas operacionais após a reforma, para o volume mensal repassado de $\mathrm{R} \$$ 73,8 milhões, $7,7 \%$ ou $\mathrm{R} \$ 5.700 .000$ poderiam ser diminuídos sem prejuízo do desempenho desses hospitais. Esses dados trazem referências para os itens a serem contemplados nos contratos de metas, por hospital, além de mostrarem qual o montante de volume financeiro a ser liberado pelo nível federal.

A Tabela 4 utiliza a média e mediana de indicadores de intensidade e de dedicação de ensino para os hospitais eficientes e ineficientes, mostrando uma tendência dos hospitais eficientes, modelados por variáveis da dimensão assistencial, também comportarem uma maior carga de atividades na dimensão de ensino.

Tabela 4. Intensidade e dedicação de ensino de acordo com a eficiência hospitalar. Brasil, 2003-2006.

\begin{tabular}{lcccc}
\hline Eficiência & $\begin{array}{c}\text { Média } \\
(\mathrm{DP})\end{array}$ & Mediana & $\begin{array}{c}\text { Média } \\
(\mathrm{DP})\end{array}$ & Mediana \\
\hline Eficiente & $0,52(0,21)$ & 0,51 & $0,63(0,29)$ & 0,58 \\
Ineficiente & $0,39(0,20)$ & 0,37 & $0,43(0,29)$ & 0,37 \\
\hline
\end{tabular}

Fonte: SIHUF/2006 


\section{DISCUSSÃO}

O Índice de Malmquist calculado por meio de DEA tem sido utilizado para avaliação de reformas de sistemas de financiamento hospitalar, tais como as que ocorreram na Finlândia e Áustria, em 1993 e 1997, respectivamente. ${ }^{8,17}$ Uma das principais vantagens da aplicação de DEA para identificação da fronteira de eficiência está no fato de que as medidas de mudanças de produtividade podem ser analisadas a partir de dois componentes distintos: as "mudanças na tecnologia" (decorrente do deslocamento da fronteira no tempo) e as "mudanças na eficiência técnica" (desempenho comparativo da unidade analisada). Nos referidos estudos, houve acompanhamento dos resultados por, no mínimo, quatro anos consecutivos e, em ambos os casos, observou-se melhora expressiva da fronteira tecnológica nos anos que sucederam a reforma, sem alteração significativa na eficiência técnica dos hospitais.

No presente estudo, de forma contrária, observou-se a melhoria evolutiva da eficiência técnica dos hospitais de ensino, com aumento de $55 \%$ das unidades localizadas na fronteira de eficiência após a maior injeção de recursos financeiros. Todavia, o mesmo não ocorreu com a mudança tecnológica, entendida como o novo modelo baseado na gestão orçamentária fixa dos hospitais. No entanto, ainda é cedo para se traçar um diagnóstico negativo para a reforma proposta, uma vez que está em processo de implementação, e o contrato de gestão, como instrumento de pactuação, ainda está em fase de desenvolvimento, principalmente no que tange às metas de ensino e de pesquisa. O presente estudo, portanto, prescinde de acompanhamento por um período maior para que se conclua pelo acerto ou não do novo modelo de financiamento. Como a modelagem somente considerou os hospitais gerenciados pelo MEC, outros estudos devem abarcar a totalidade de hospitais de ensino, com as suas variadas naturezas jurídicas, para verificar se há replicação desses resultados.

Dada a intensidade da crise de financiamento no período precedente à reforma, $\mathrm{o}$ aumento dos recursos implicou a minimização da distância de deslocamento e/ou de contração da fronteira e o alívio financeiro para os hospitais de ensino que, inclusive, tiveram estratégias diferenciadas para gerenciarem a produtividade (observado pelas alterações no volume de internações, cirurgias e consultas ambulatoriais da Tabela 1). Aqueles hospitais que, apesar do aumento dos recursos, ainda tiveram queda na eficiência técnica, seriam objetos de estudo sobre a capacidade de gestão local da produção. Nesses casos, a avaliação dos hospitais de referência, conforme mostrado na Tabela 3, pode ser um caminho viável para a busca de sua melhoria gerencial. Essa informação tem utilidade tanto para a unidade em si, ao orientar quais os seus próprios benchmarks, quanto para o órgão regulador, ao identificar possíveis metas a serem pactuadas e ao criar parâmetros de ajuste do volume de recursos aos produtos esperados. ${ }^{9}$

Ainda, a eficiência da produtividade assistencial pode estar associada à qualidade das atividades de ensino desenvolvidas pelos hospitais. Apesar de as atividades de ensino estarem relacionadas ao aumento do custo da produção assistencial nos hospitais, ${ }^{6}{ }^{\circ}$ desenvolvimento e a excelência do ensino e da pesquisa nos hospitais podem ser considerados como estratégia prioritária para o enfrentamento da crise de financiamento dos hospitais de ensino, justamente ao explorar aquilo que verdadeiramente os diferencia dos demais hospitais do País. 


\section{REFERÊNCIAS}

1. Banker RD, Charnes A, Cooper WW. Some models for estimating technical and scale inefficiencies in data envelopment analysis. Manage Sci. 1984;30(9):107892. DOI: $10.1287 / \mathrm{mnsc}$.30.9.1078

2. Caves DW, Christensen LR, Diewert WE. Multilateral comparisons of output, input and productivity using superlative index numbers. ECon J. 1982;92(365):7386. DOI: $10.2307 / 2232257$

3. Coelli TJ, Prasada RDS, Battese GE. An introduction to efficiency and productivity analysis. Boston: Kluwer; 1998.

4. Fare R, Grosskopf S, Norris M, Zhang Z. Productivity growth, technical progress, and efficiency change in industrialized countries. Am Econ Rev. 1994;84(1):66-83.

5. Farrell MJ. The measurement of productive efficiency. J R Statl Soc (Ser A). 1957;120(3):253-81.

6. Grosskopf S, Margaritis D, Valdmanis V. The effects of teaching on hospital productivity. Socioecon Plann Sci. 2001;35(3):189-204. DOI: 10.1016/S00380121(01)00006-4

7. Grosskopf S, Margaritis D, Valdmanis V. Competitive effects on teaching hospitals. Eur J Oper Res. 2004;154(11):515-25. DOI: 10.1016/S03772217(03)00185-1

8. Linna M. Health care financing reform and the productivity change in Finnish hospitals. J Health Care Finance. 2000;26(3):83-100.

9. Lins MPE, Lobo MSC, Silva AM, Fiszman R, Ribeiro VJP. O uso da Análise Envoltória de Dados (DEA) para avaliação de hospitais universitários brasileiros. Cienc Saude Colet. 2007;12(4):985-98. DOI: 10.1590/ S1413-8123200700040002010
10. Lobo MSC, Bloch KV, Fiszman R, Oliveira MR, Ribeiro VJP. Sistema de Informações dos Hospitais Universitários (SIHUF/MEC): um banco de dados administrativo. Cad Saude Colet. 2006;14(1):149-62.

11. Machado SP, Kruchenbecker R. Desafios e perspectivas futuras dos hospitais universitários do Brasil. Cienc Saude Colet. 2007;12(4):871-7. DOI: 10.1590/S141381232007000400009

12. Malmquist $\mathrm{S}$. Index numbers and indifference surfaces. Trab Estad. 1953;4:209-42.

13. McCallion G, Glass JC, Jackson R, Kerr CA, McKillop DG. Investigating productivity change and hospital size: a nonparametric frontier approach. Applied Econ. 2000;32(2):161-74. DOI: 10.1080/000368400322859

14. O’Neill LO, Rauner M, Heidenberger K, Kraus M. A cross-national comparison and taxonomy of DEA-based hospital efficiency studies. Socioecon Plann Scien. 2008;42(3):158-89. DOI: 10.1016/j. seps.2007.03.001

15. Ozcan, Y. Sensitivity analysis of hospital efficiency under alternative output/input and peer group: a review. Knowledge and policy. Int J Knowl Transf Util. 1992;5(4):1-29.

16. Sahin I, Ozcan YA. Public sector hospitals efficiency for provincial markets in Turkey. J Med Syst. 2000;24(6):307-20. DOI: 10.1023/A:1005576009257

17. Sommersguter-Reichmann M. The impact of the Austrian hospital financing reform on hospital productivity: empirical evidence on efficiency and technology changes using a non-parametric inputbased Malmquist approach. Health Care Manag Scien. 2000;3(4):309-21. DOI: 10.1023/A:1019022230731 